\title{
ESCRIBIR TEXTOS ARGUMENTATIVOS DESDE EL INICIO DE LA ESCOLARIDAD. UN ANÁLISIS DE TEXTOS PRODUCIDOS A PARTIR DE UNA SECUENCIA DIDÁCTICA
}

\section{WRITING ARGUMENTATIVE TEXTS SINCE THE BEGINNING OF SCHOOL. AN ANALYSIS OF WRITTEN TEXTS PRODUCED WITH A DIDACTIC SEQUENCE}

\author{
Verónica Sanchez Abchi* \\ Joaquim Dolz \\ Ana María Borzone
}

\section{RESUMEN}

Este trabajo analiza los textos argumentativos escritos por alumnos de $3^{\circ}$ grado de primaria, de Córdoba (Argentina), producidos a partir de una secuencia didáctica para el aprendizaje del género "carta de solicitud". El estudio explora, además, el impacto del dispositivo didáctico en el desempeño de los alumnos. Para el diseño del instrumento y el análisis de las producciones nos basamos en los principios del interaccionismo sociodiscursivo (Bronckart, 2004). Los resultados mostraron que los niños avanzaban en la situación comunicativa, la planificación y el formato de la carta y mejoraban sus capacidades argumentativas. Se discuten, por último, las implicancias pedagógicas del trabajo.

Palabras-llave: texto argumentativo; escritura de textos; secuencia didáctica; interaccionismo sociodiscursivo.

\section{ABSTRACT}

This work analyzes written argumentative texts produced by children from 3rd year of primary education from Córdoba (Argentina), in the frame of the application of a didactic sequence, designed to write formal request letters. The study examines, as well, the impact of the sequence in the children's performance. The elaboration of the instrument and the analyses of the productions were based on the principles of the Socio-discursive Interactionism (Bronckart, 2004). The results showed that the children improved their

\footnotetext{
"CONICET, Buenos Aires, Argentina. vssancheza@yahoo.com.ar $r_{i}$ Universidad de Ginebra, Genebra, Suiça. Joaquim.Dolz-Mestre@unige.ch; CONICET, Buenos Aires, Argentina. anaborzone44@ gmail.com
} 
argumentative skills and progressed in their comprehension of the communicative situation, the planification process and the formal conventions of the letter. The pedagogical implications are discussed.

Keywords: argumentative texts; text writing; didactic sequence; sociodiscursive interactionism.

\section{INTRODUCCIÓN}

Este trabajo tiene por objeto analizar los textos argumentativos escritos por alumnos de $3^{\circ}$ de primaria, de escuelas públicas de Córdoba (Argentina). Los textos fueron producidos en el marco de una secuencia didáctica para el aprendizaje del género "carta de solicitud". En la consigna de producción se solicitaba a los alumnos que justificaran con argumentos simples su pedido.

Aprender a argumentar, en el sentido de desarrollar un espíritu crítico frente a los valores de la sociedad y apropiarse de los mecanismos de la lengua para defender o discutir una postura, constituye un contenido fundamental para la formación de ciudadanos capaces de opinar, participar y construir una sociedad democrática y plural (CAMPS y DOLZ, 1995). En la escuela, la argumentación no se limita al área de lengua, sino que puede presentarse también en textos de otras disciplinas del currículo escolar. Es en este sentido que resulta necesaria la implementación y evaluación de herramientas que promuevan su aprendizaje.

En Argentina, los programas oficiales proponen un trabajo abordando la diversidad textual desde el inicio de la escolaridad (CBC, Núcleos de Aprendizaje prioritario ${ }^{1}$ ). Sin embargo, tradicionalmente, la escuela primaria ha priorizado la enseñanza del texto narrativo; mientras que otros tipos textuales, como el expositivo o el argumentativo tienen escasa presencia en el aula (SÁNCHEZ ABCHI, 2009). Los programas no dan precisiones sobre cómo abordar la argumentación y, en la práctica, se asocia a la enseñanza de la lengua oral y ocupa un lugar marginal en los programas. Asimismo, se aborda tardíamente en la escuela, porque se considera un texto complejo y difícil para los alumnos (HOCEVAR, 2007), situación observada también en el contexto educativo de otros países (CAMPS y DOLZ, 1995).

La argumentación, no obstante, constituye una conducta lingüística efectiva de los niños, fuertemente ligada a los procesos de socialización y desarrollo cognitivo (BRASSART, 1995). Los niños pueden elaborar argumentos simples desde edades

1 Los "CBC" - Contenidos Básicos Comunes- y los Núcleos de Aprendizaje Prioritario son documentos propuestos por el Ministerio de Educación de Argentina, para orientar las líneas de enseñanza definidas por los programas escolares. 
tempranas y ya desde los tres años son capaces de considerar al interlocutor a fin de alcanzar un objetivo (GOLDER, 1996). Asimismo, en situaciones polémicas, los niños pequeños pueden hacer uso de estrategias argumentativas más complejas (SILVA, 2002). Sin embargo, la producción escrita de la argumentación razonada o la argumentación clásica de tradición greco latina, - que constituye el género tradicionalmente escolarizado- plantea dificultades aún en niveles avanzados de la escolaridad. En español, los estudios dan cuenta de dificultades que presentan niños y jóvenes, en la producción de textos argumentativos, relacionados con la superestructura de los textos, el dominio de operaciones de textualización, la capacidad de descentración, la incorporación de otras voces, la producción de argumentos circulares, la elaboración de conclusiones demasiado generales o el aprendizaje tardío de estrategias de contra-argumentación o refutación (ARROSSI, AXELRUD, AGOSTINO \& EISNER, 2002; GARCÍA JURADO, LÓPEZ GARCÍA \& MIÑONES, 2002; NUÑEZ LAGOS, 1999; PARODI SWEIS, 2000; SILVESTRI, 2001; entre otros). Es importante aclarar que estos trabajos no consideran el efecto de la enseñanza en la producción, por lo que no sabemos si las dificultades citadas podrían superarse en el marco de una intervención.

Los discursos argumentativos requieren una enseñanza específica, que considere tanto los aspectos comunicativos, como los aspectos ligados a la estructura y organización de las producciones y los mecanismos de textualización (BRASSART, 1995). El aprendizaje del texto argumentativo, no necesariamente posterior al del texto narrativo, puede promoverse de manera precoz, y en paralelo con otros tipos textuales, si se utilizan instrumentos adecuados de enseñanza (BRASSART, 1995; DOLZ, 2001; 1996; 1995; DOLZ, MABILLARD, COUCHEPIN TOBOLA \& VEUILLET, 2009; COTTERON, 1995; FELTON, 2004; KUHN \& UDELL, 2003).

En el marco de estos planteos, nos hemos propuesto el diseño, la implementación y la evaluación de una secuencia didáctica de textos argumentativos para el primer ciclo de la escuela primaria, puntualmente, la producción escrita de cartas de solicitud, con justificación.

En este marco, nos hemos planteado un doble propósito: a) en primer lugar, analizar las producciones escritas de los alumnos de $3^{\circ}$ grado de primaria, producidas en el marco de una secuencia didáctica para la enseñanza del género "carta de solicitud con justificación", a fin de identificar los conocimientos de los alumnos, sus dificultades y el nivel de dominio sobre los componentes del género; b) en segundo lugar, evaluar la incidencia del dispositivo didáctico en el aprendizaje 
de los alumnos, esto es, identificar los aspectos en los que se observan avances a partir de la implementación de las actividades.

Para la elaboración de las secuencias y el análisis de los textos producidos a partir de este dispositivo, nos hemos basado en los principios del interaccionismo sociodiscursivo -en adelante ISD- (BRONCKART, 2004; BRONCKART, BAIN, SCHNEUWLY, DAVAUD y PASQUIER, 1985). En los apartados siguientes, desarrollamos los principios teóricos que han guiado la concepción del trabajo.

\section{El interaccionismo sociodiscursivo}

El ISD concibe los textos como secuencias de unidades lingüísticas en los que los parámetros contextuales intervienen como fuente de las acciones lingüísticas. Se describe el proceso de producción a través de tres tipos de operaciones: en primer lugar, aquellas relacionadas con la base de orientación contextual, que otorgan, precisamente, una base general para la producción, resultado de una representación interna del contexto social y material de la actividad verbal, que interviene en la producción de un género textual (SCHNEUWLY, 1988). Supone la fijación de variables o parámetros comunicativos relacionados con la interacción social, que remiten a la definición del lugar social, a la precisión del objetivo de la situación comunicativa y a la relación que se establece entre el enunciador y el destinatario. En segundo término, las operaciones de estructuración suponen la selección del género y el proceso de planificación. Por último, las operaciones de textualización abordan, en un nivel local, la construcción de la cadena textual. Los medios disponibles para marcar las relaciones entre enunciados se articulan en torno a tres dominios: la cohesión, la conexión/ segmentación y la modalización. La primera hace referencia a la organización de las unidades de contenido en una sucesión ininterrupida, a fin de asegurar la continuidad y la progresión textual. Se relaciona con la sucesión de los sintagmas nominales y verbales. La conexión/ segmentación se sustenta en el sistema de conectores y de marcas de puntuación, mecanismos estos que permiten a la vez segmentar el discurso y articular los núcleos predicativos sucesivos (FAYOL Y SCHNEUWLY, 1988). Las operaciones postuladas permiten comprender las unidades lingüísticas de un texto como huellas que resultan de la acción lingüística en un contexto dado. En este sentido, se plantea que el ISD se inscribe en la psicología del lenguaje y en la teoría socio histórico cultural (VIGOTSKY, 1988). 


\section{Las secuencias didácticas}

Desde la perspectiva del ISD, se han desarrollado "secuencias didácticas", instrumentos para la enseñanza y el aprendizaje de diferentes géneros textuales. Las secuencias constituyen un dispositivo que posibilita la apropiación de estrategias de producción del género en la escuela, permite esclarecer los contenidos y determina los objetivos de enseñanza en relación con la situación comunicativa, el género textual y los mecanismos lingüísticos involucrados. La SD agrupa un conjunto de tareas o ejercicios al servicio de la producción de un género, dentro de un proyecto de clases. Las actividades se organizan en módulos que abordan, de manera específica, y en círculos de complejidad creciente, aspectos del género textual sobre el que se trabaja (DOLZ \& SCHNEUWLY, 1998). En una primera fase -la "puesta en situación" -, se plantean los objetivos de la producción textual a través de la consigna y se definen los participantes de la situación. En el contexto escolar, la tarea planteada se entiende como una situación ficcional que ayuda a los niños a contextualizar su producción. Esta "ficcionalización" permite sentar las bases de orientación contextual para producir los textos en el aula (SCHNEUWLY \& DOLZ, 1997). Como resultado de esta primera fase, los alumnos escriben una primera versión del texto. Se trabajan luego aspectos puntuales del género propuesto $\mathrm{y}$, finalmente, tiene lugar una instancia de revisión y de nueva producción textual (COTTERON, 1995; DOLZ Y PASQUIER, 1993 A; 1993 B; DOLZ, NOVERRAZ Y SCHNEUWLY, 2001).

Durante la década de los noventa, en la Universidad de Ginebra, se llevaron a cabo investigaciones que observaron un efecto positivo de las secuencias en la producción textual escrita de los alumnos (cf. Dolz y Schneuwly, 1996). Los resultados de las investigaciones mostraron que los niños que participaron en experiencias de intervención con secuencias didácticas realizaron importantes avances en relación con las habilidades para apoyar argumentos, considerar al destinatario, negociar frente a una eventual contradicción y utilizar unidades lingüísticas específicas de los textos argumentativos -como organizadores textuales de causa y de conclusión, modalizadores de probabilidad, certeza y concesión y fórmulas de cortesía- (DOLZ, 1996, 1995). Sin embargo, se han observado dificultades relacionadas con otros aspectos de la producción. Cuando en el aula se propone una tarea de escritura con un género de circulación social, hablamos de géneros "escolarizados" que constituyen una suerte de ficcionalización de los géneros de referencia de circulación social, lo que da lugar al término "géneros escolarizados" (SCHNEUWLY \& DOLZ, 1997). Comprender el carácter ficcional 
de los géneros escolarizados puede presentar dificultades tanto para los alumnos como para los docentes (VUILLET, MABILLARD, TOBOLA \& DOLZ, en prensa).

Si bien la enseñanza de la lengua a través del género ha sido desarrollada más extensamente en francés, también se han llevado a cabo experiencias en otras lenguas (CAMPS, 2003; DOLZ Y PASQUIER, 2000, IDIAZABAL \& LARRIGAL, 1997; SÁINZ, 2000; SANTAMARIA, 2003 entre otros). En Argentina, en cambio, el diseño y la implementación de secuencias didácticas no han tenido mayor difusión.

En este marco, el presente trabajo se propone, entonces, analizar las producciones de los niños producidos en el marco de la secuencia y evaluar el impacto del dispositivo en el aprendizaje de los niños.

\section{METODOLOGÍA}

\subsection{Participantes}

Participaron de este estudio 2 docentes y 62 alumnos de $3^{\circ}$ grado de una escuela primaria de la provincia de Córdoba, Argentina. El grupo de alumnos estaba formado por 25 niños y 37 niñas, que asistían a dos secciones de tercer grado, de una escuela pública urbana (edad promedio de 8.04 ; extremos 7.6 y 9.7). Provenían de familias de clase media y media baja. Un 36\% de los padres había completado el secundario y un $13 \%$ había seguido o comenzado estudios terciarios o universitarios.

\subsection{Secuencia didáctica}

La secuencia didáctica elaborada para este experimento es una adaptación, en español (SÁNCHEZ ABCHI \& BORZONE, 2010) de materiales didácticos publicados en la Suiza francófona (DOLZ, NOVERRAZ Y SCHNEUWLY, 2001). Las actividades se diseñaron considerando el contexto de trabajo y los aportes de las docentes participantes (ver procedimiento). La secuencia se desarrolló a lo largo de 20 sesiones - de entre 15 y 45 minutos- organizadas en módulos temáticos que se distribuyeron en tres semanas de clase. La tabla 1 resume las actividades de cada módulo. 
Cuadro I. Secuencia didáctica para la producción de cartas de solicitud

\begin{tabular}{|c|c|c|}
\hline \multicolumn{3}{|c|}{ PRODUCCIÓN INICIAL } \\
\hline MODULO & OBJETIVO & ACTIVIDADES \\
\hline 1 & $\begin{array}{l}\text { - Reconocimiento de carta } \\
\text { entre otros tipos de } \\
\text { textos. } \\
\text { Identificación del formato } \\
\text { de una carta. }\end{array}$ & $\begin{array}{l}\text { - Comprensión y contraste de las ca- } \\
\text { racterísticas de un cuento y de una car- } \\
\text { ta de solicitud. } \\
\text { - Lectura de cartas e identificación de } \\
\text { tema y destinatario. } \\
\text { - Presentación del formato. } \\
\text { - Reconocimiento de las diferentes } \\
\text { partes de las cartas. } \\
\text { - Actividad de puzzle con cartas des- } \\
\text { organizadas. } \\
\text { - Sistematización y repaso. }\end{array}$ \\
\hline 2 & $\begin{array}{l}\text { - Identificación y } \\
\text { Producción de solicitudes. }\end{array}$ & $\begin{array}{l}\text { - Lectura de cartas para identificar las } \\
\text { solicitudes. } \\
\text { - Producción de solicitudes orales. } \\
\text { Dramatización. } \\
\text { - Identificación y reformulación de } \\
\text { solicitudes en textos } \\
\text { - Reconocimiento de diferentes expre- } \\
\text { siones para la solicitud y de fórmulas } \\
\text { de cortesía. } \\
\text { - Completamiento de solicitudes. } \\
\text { - Producción oral de solicitudes. Dra- } \\
\text { matización. } \\
\text { - Reformulación oral y escrita de so- } \\
\text { licitudes. } \\
\text { - Sistematización y repaso. }\end{array}$ \\
\hline 3 & $\begin{array}{l}\text { - Identificación de } \\
\text { justificación de un pedido } \\
\text { y de conectores de causa. } \\
\text { Producción de } \\
\text { justificaciones orales y } \\
\text { escritas. }\end{array}$ & $\begin{array}{l}\text { - Producción oral de justificaciones. } \\
\text { Dramatización. } \\
\text { - Producción escrita de solicitudes y } \\
\text { justificaciones. } \\
\text { - Identificación de la justificación en } \\
\text { textos. } \\
\text { - Completamiento de solicitudes con } \\
\text { la justificación correspondiente. Traba- } \\
\text { jo a partir del conector "porque". } \\
\text { - Identificación de la pertinencia de las } \\
\text { justificaciones. }\end{array}$ \\
\hline
\end{tabular}




\begin{tabular}{|l|l|l|}
\hline 4 & $\begin{array}{l}\text { Identificación y empleo de } \\
\text { signos. }\end{array}$ & $\begin{array}{l}\text { - Completamiento de signos de pun- } \\
\text { tuación de cartas. }\end{array}$ \\
\hline 5 & $\begin{array}{l}\text { Práctica de revisión. } \\
\text { PRODUCCIÓN FINAL }\end{array}$ \\
\hline \multicolumn{2}{|c|}{} \\
\hline
\end{tabular}

\subsection{Procedimiento}

Las docentes participaron en dos sesiones de formación previas a la intervención, en las que se explicaron los principios y objetivos del trabajo en secuencias y el abordaje por el género, se discutieron las actividades propuestas y su implementación y se consensuaron las consignas, atendiendo a los contenidos curriculares y a las planificaciones áulicas. Las docentes fueron las encargadas de llevar a cabo las actividades en el aula.

La consigna fue la siguiente: "vamos a escribir una carta al 'señor director del zoológico' para pedirle visitar el zoológico. Le vamos a explicar por qué queremos visitarlo." La consigna fue la misma para la producción inicial y final.

Durante la intervención, se realizaron reuniones semanales con las docentes para analizar las actividades realizadas, planificar tareas y acordar criterios. Se realizaron también observaciones y registros de clases para el seguimiento del trabajo en el aula. Para el análisis se consideraron los textos producidos antes de la aplicación de la secuencia o producción inicial y después -producción final- (En adelante, PI y PF, respectivamente).

\subsection{Análisis de la información empírica}

El corpus de análisis estuvo constituido por 124 textos (62 en cada momento de producción). Los textos fueron transcriptos para el análisis, que se realizó de acuerdo a las siguientes categorías:

a) Extensión de las producciones medida en cantidad de palabras.

b) Adaptación a la situación comunicativa. En el contexto áulico, las bases de orientación contextual son definidas por la consigna planteada (DOLZ, GAGNON \& TOULOU, 2008). Para analizar este aspecto, se consideró el nivel de adecuación a la consigna. 
c) Formato. Se atendió al dominio de las convenciones de la carta: indicaciones de lugar y fecha, encabezamiento, saludos y firma, organización y disposición gráfica en la página.

d) Contextualización de la solicitud. Se consideró si los niños se presentaban a sí mismos y al proyecto escolar y si explicaban el contexto de su pedido.

e) Contenido. Se tuvieron en cuenta los temas tratados en las producciones.

f) Planificación argumentativa. Se atendió a la manera en que la solicitud y los argumentos se organizaban y se presentaban en el texto. Se consideró tanto la cantidad de argumentos como su organización en la estructura discursiva.

g) Textualización. Se atendió al empleo de organizadores textuales, la puntuación, la modalización en la solicitud (empleo del imperfecto de cortesía, imperfecto de subjuntivo, subordinada interrogativa indirecta, etc.) y fórmulas de cortesía.

\section{RESULTADOS}

Si bien participaron de este estudio dos grupos diferentes, los resultados se presentan de manera conjunta, porque ambos grupos participaron de la misma intervención y las producciones, tanto iniciales como finales, se asemejan en las fortalezas y obstáculos observados.

\subsection{Extensión de las producciones}

El análisis de las producciones finales permitió identificar avances en la escritura. En primer lugar, se advierte un incremento de la extensión, medida en cantidad de palabras, que pasa de un promedio de 42.92 en la PI a 50.47 en la final. Los textos, muy escuetos en la PI, avanzan en la extensión de manera significativa, como lo demuestran los test estadísticos realizados $(t=-2,755, \mathrm{p}<.05)^{2}$.

\subsection{Adaptación a la situación comunicativa y adecuación a la consigna.}

En este apartado, que considera la adecuación de los textos como respuesta a la consigna, el análisis muestra importantes diferencias entre las producciones iniciales y finales.

Antes de la intervención, el 70,9\% de los textos responde de manera completa a la consigna, mientras que un 14,5\% plantea la solicitud sin argumentos. Los textos restantes no presentan la solicitud: uno de ellos es un diálogo ficticio con el director

2 La prueba realizada muestra que la diferencia entre los resultados de la PI y la PF es significativa porque es menor a .05 . 
del zoológico, mientras que otros son cartas con recomendaciones para el cuidado de los animales, la expresión de deseos o gustos personales o la notificación de la visita.

En la PF, el porcentaje de adecuación a la consigna alcanza el 79\%; mientras que, en dos textos $(3,2 \%)$ la solicitud parece involuntariamente omitida y en otros siete $(11,2 \%)$ se realiza el pedido pero no se lo justifica. En cuanto a las cartas que no responden en absoluto a la consigna $(6,6 \%)$, es necesario realizar una salvedad sobre la "ficcionalización" que propone la consigna y que permite sentar las bases de orientación contextual para producir los textos en el aula. En nuestro trabajo, el proceso de ficcionalización de la consigna sigue un recorrido particular: en el momento de escribir la PF, las maestras les habían ya confirmado a los alumnos la fecha de visita al zoológico. Esta información incide en las producciones de los alumnos, porque la situación ficcional planteada por la consigna se modifica. En cuatro casos, las cartas manifiestan la alegría por realizar la visita, puesto que saben que es un hecho y la consigna original no representa parámetros comunicativos válidos para los alumnos. En este sentido, estas producciones no reflejan problemas para comprender la situación comunicativa real -la visita ya confirmada- y producir un texto adecuado a dicha situación. El problema radica en que los alumnos no se apropian del carácter ficcional de la consigna, lo que modifica el proceso de planificación textual. Más allá de esta salvedad, los datos muestran que el grupo avanza de manera explícita en su comprensión de la situación comunicativa, lo que puede considerarse resultado de la intervención pedagógica implementada.

\subsection{Formato del género "carta de solicitud"}

Con respecto al formato de la carta, los progresos son notables. En las producciones iniciales, sólo dos textos respetan, parcialmente, el formato; mientras que los demás textos presentan problemas diversos. En la mayoría de las cartas se explicita el destinatario, pero no se lo ubica espacialmente de manera adecuada o no se lo introduce convencionalmente: "de Karen para el mejor director del zoológico del mundo". Por otra parte, la firma y las referencias de lugar tienen escasa presencia. No hay divisiones de párrafos ni fórmulas de introducción o cierre; no obstante, algunas cartas incluyen saludos con características coloquiales. En las producciones finales, en cambio, en el $95 \%$ de las producciones se respeta el formato y en un $72 \%$ se reconocen párrafos.

En tanto que la distribución en párrafos puede asociarse a la organización de la información, el progreso en este aspecto podría ser resultado del avance en 
la planificación textual. Si bien el formato de la carta es un aspecto formal que se aprende con relativa facilidad (BRASSART, 1995), las diferencias entre PI y PF resultan muy marcadas y es claro el impacto de la secuencia didáctica en los progresos observados.

\subsection{Contextualización de la solicitud.}

En ningún texto ni de la PI ni de la PF se contextualizó la solicitud. Esto es, los alumnos no se presentaban a sí mismos, no explicaban la situación comunicativa ni el por qué de la visita al zoológico. Esto puede vincularse directamente con las prácticas en el aula. Solo una de las docentes, en su interacción oral, contextualizó fuertemente la propuesta de escritura. Pero en ningún caso la presentación del autor o de la situación fue parte de la consigna presentada a los alumnos.

\subsection{Contenido}

El proceso de planificación supone la generación de ideas, la selección de la información que resulta pertinente para la producción y la organización de los contenidos de acuerdo con los objetivos, la intención comunicativa y el género textual (FAYOL y SCHNEUWLY, 1987). En este apartado se analiza la selección de contenidos y su organización, que se encuentra ligada a la planificación argumentativa, como veremos en el apartado siguiente.

El análisis muestra que los textos de la PI son breves y se centran - salvo aquellos que no cumplen la consigna- sobre un único tema: la solicitud. Los tópicos de los argumentos que apoyan el pedido son los gustos personales y las actividades escolares, como muestra el ejemplo:

(1) Nos podría dejar entrar a ver su zoológico Para ver los animales porque estamos trabajando con los animales

La selección de los tópicos resulta esperable porque la solicitud está ligada al contexto de la escuela y porque es común que los niños de esta edad apelen a sus propias experiencias para justificar sus posiciones (GOLDER, 1996).

Varios textos muestran problemas de organización, porque la presentación se encuentra en el medio o al final del texto o reiteran la solicitud, intercalada con argumentos (14 casos). En algunas cartas se abordan también otros contenidos, como comentarios sobre los animales o el relato de experiencias personales, que, lejos de apoyar la solicitud, parecen desconectados de la argumentación. 
En la diversidad de los patrones de organización observados se pudo identificar, aunque con un porcentaje relativamente bajo, una estructura predominante: a) apelación al destinatario, b) solicitud y c) uno o más argumentos. Esta estructura se observó en $29.03 \%$ de los textos.

En las producciones finales, la estructura se complejiza: a) apelación al destinatario, b) eventualmente una presentación, c) solicitud, d) uno o más argumentos, e) cierre-que puede ser un agradecimiento, una expresión de deseo de que se realice la visita o un saludo-. Al mismo tiempo, la cantidad de producciones con este esquema se incrementa hasta un $58,06 \%$, lo que supone un aumento notable respecto de los textos bien estructurados en la PI. La introducción de otros contenidos -como comentarios sobre los animales o el relato de experiencias personales- apoya la solicitud y se incluyen, además, ejemplos y enumeraciones, como parte del pedido o la argumentación. Los datos nos muestran una tendencia a organizar mejor los textos y a seleccionar con mayor pertinencia la información que se incluye.

Por otra parte, se observa que algunas producciones (8\%) incluyen contenidos no relacionados con las convenciones del género carta de solicitud, como la introducción de un vocabulario técnico propio de las ciencias naturales:

(2) Señor director podemos ver los animales ovíparo y vivíparo los que nacen de la panzas y de los buevos $(\mathrm{PI})$

El ejemplo sugiere la influencia de otros géneros textuales típicamente escolares, como el texto informativo, y revela una dificultad de los alumnos que podría asociarse con la apropiación de la consigna. En la escuela, la situación de "escritura de un texto en hoja aparte", está tradicionalmente asociada a la evaluación. La inclusión de rasgos de un género típicamente escolar en una carta de solicitud podría vincularse con la necesidad del niño de "escolarizar" una actividad que, en principio, quiere presentarse como auténtica. En este proceso, los alumnos quieren mostrar que han incorporado otros contenidos escolares. La dificultad para asumir los parámetros comunicativos presentados en la consigna y la asimilación de la tarea a una actividad escolar se convierten en obstáculos para el abordaje y la comprensión del género.

\subsection{Planificación argumentativa}

En el análisis de la planificación argumentativa, puntualmente, se consideran tanto la cantidad de argumentos como su organización en la estructura discursiva. 
En las producciones, se observa una preeminencia de argumentos encabezados con la preposición "para" más infinitivo. La argumentación parece "amalgamada" con la solicitud: se encuentran ligadas sintácticamente y se formulan de manera conjunta. En menor medida se observan argumentos introducidos por el organizador "porque" o "así que". Algunos alumnos apelan a otro tipo de argumentos, como proposiciones condicionales ("si nos dejas ir voy a estar muy contento") o la promesa de un buen comportamiento ("te prometo que nos vamos a portar bien").

El análisis permitió discriminar cuatro grupos de textos, de acuerdo a la cantidad y organización de los argumentos: a) textos con argumentación amalgamada a la solicitud, introducida por la preposición "para" + infinitivo; b) textos con un argumento simple $;$ c) textos que presentan o bien la finalidad amalgamada más un segundo argumento, o bien dos argumentos que pueden deslindarse claramente de la demanda; d) textos con más de dos argumentos. El gráfico sintetiza los resultados generales.

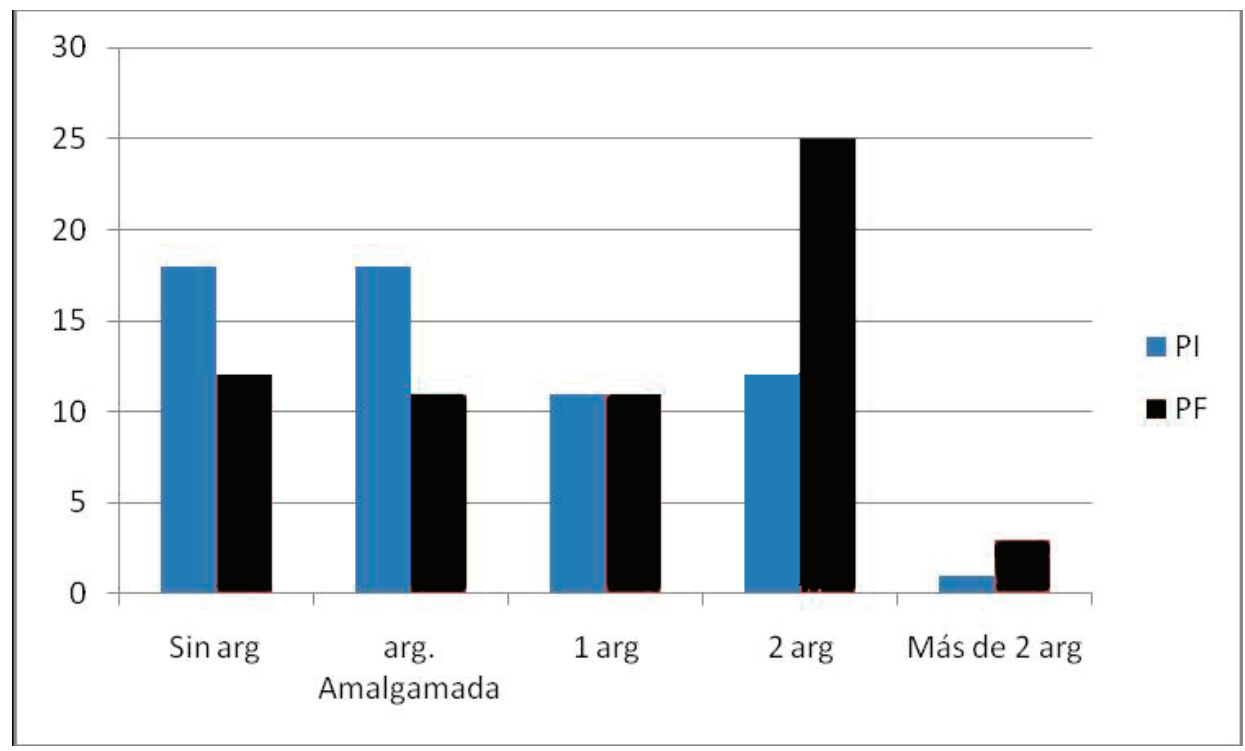

Figura I. Planificación argumentativa en la PI y PF

El patrón de planificación se modifica entre ambas producciones y la correlación entre la PI o PF y la estructura argumentativa resulta estadísticamente significativa (coeficiente de correlación .223, $\mathrm{p}=<.005$ ). En la tabla 1 se presenta el porcentaje de textos por categoría. 
Tabla 1. Planificación argumentativa

\begin{tabular}{|l|c|c|}
\hline \multicolumn{1}{|c|}{ Categorías } & PI & PF \\
\hline \multicolumn{1}{|c|}{ Sin argumento } & 29.03 & 19.35 \\
\hline A. Argumentación amalgamada & 29.03 & 17.74 \\
\hline B. Un argumento simple & 17.74 & 17.74 \\
\hline C. Dos argumentos & 19.35 & 40.32 \\
\hline D. Más de dos argumentos & 1.61 & 4.84 \\
\hline
\end{tabular}

En las producciones finales se advierte una tendencia a una planificación argumentativa más compleja. Se reduce la cantidad de textos sin argumentos y con argumentación amalgamada; se incrementa el número de producciones con dos o más justificaciones y se eleva la media de argumentos por textos (PI: Media $=1$; PF Media $=1.34$ )

Esta tendencia podría reflejar una progresión de un proceso de planificación online $^{3}$ a una incipiente planificación más elaborada. Desde el punto de vista didáctico, nos preguntamos qué aspectos de la intervención pudieron haber incidido en este avance. Por una parte, en las clases se hizo una constante referencia a la necesidad de convencer a los destinatarios, por lo que el progreso en la argumentación podría asociarse a una mayor conciencia de los parámetros comunicativos. Por otra parte, en la secuencia se realizaron actividades de producción de argumentos.

Sin embargo, si se considera que los avances son moderados, es posible pensar que el trabajo tuvo limitaciones. Las actividades desarrolladas fueron generales y no explícitamente aplicadas al género "carta de solicitud". Las docentes no trabajaron, puntualmente, sobre argumentos que los niños podían usar para apoyar sus pedidos en la carta y que podían ayudarlos a mejorar la producción. Esto resulta coincidente con otras experiencias de aplicación de las secuencias en la suiza francófona, en las que se observó que los maestros no siempre trabajan sobre las dificultades que presentan los alumnos (PIETRO, PFEIFFER RYTER, WIRTHNER, BEGUIN, BROI, CLÉMENT, MATEI, \& ROOS; 2009). Los resultados sugieren la necesidad de abordar la argumentación de una manera situada y contextualizada en el marco del trabajo con el género específico que se aborde en la secuencia didáctica, en este caso la carta de solicitud, en función de construir textos que apoyen de manera pertinente la solicitud.

3 La planificación on-line hace referencia a una planificación "paso a paso", sin una perspectiva general que organice la información. Este tipo de planificación es típica de los escritores inexpertos. (BEREITER \& SCARDAMALIA, 1987) 


\subsection{Textualización}

En este apartado se presenta un análisis sobre las operaciones de textualización de los alumnos. No todos los recursos lingüísticos fueron trabajados en la secuencia didáctica. Sin embargo, en tanto que se encuentran estrechamente vinculados con las demás operaciones de producción discursiva, nos parece necesario relevar las dificultades y las fortalezas de los alumnos en este aspecto.

Organizadores textuales: El empleo de organizadores textuales no fue objeto de un módulo específico en la secuencia, aunque en la interacción las docentes hacían observaciones al respecto. En este sentido, resulta esperable que entre las producciones iniciales y finales se observen diferencias mínimas. La tabla 2 presenta la cantidad de textos que utilizan los diferentes organizadores.

Tabla 2. Cantidad de textos que incluyen organizadores

\begin{tabular}{|l|c|c|}
\hline \multicolumn{3}{|c|}{ Organizadores textuales } \\
\hline & $\mathrm{PI}$ & $\mathrm{PF}$ \\
\hline Porque & $29,03 \%$ & $45,1 \%$ \\
\hline Para + infinitivo & $41,9 \%$ & $45,1 \%$ \\
\hline Asi / Así que & $11,2 \%$ & $4,8 \%$ \\
\hline Para que & $3,2 \%$ & $1,6 \%$ \\
\hline Si (condicional) & $4,8 \%$ & $19,35 \%$ \\
\hline Pero, aunque & 11,2 & $9,6 \%$ \\
\hline También / Además & $3,2 \%$ & $4,8 \%$ \\
\hline Como, Por ejemplo & $6,4 \%$ & $11,2 \%$ \\
\hline Cuando (temporal) & $3,2 \%$ & $12,9 \%$ \\
\hline Y (intraoracional) & $41,9 \%$ & $27,4 \%$ \\
\hline Textos sin organizadores & $14,5 \%$ & $3,2 \%$ \\
\hline
\end{tabular}

El progreso relativamente modesto puede atribuirse a la ausencia de actividades específicas en los módulos. El avance más importante se observa en el organizador lógico argumentativo "porque" -prototípico de la causalidad, de relativamente temprana aparición y de alta frecuencia en el español oral y escrito. Los resultados observados en el empleo de los organizadores, aun sin intervención específica, son interesantes porque puede inferirse que fue el trabajo con los contenidos lo que ejerció un efecto de atracción de estas unidades textuales. Sin embargo, el abordaje tangencial no parece suficiente para mostrar progresos que se reflejen en la organización textual. Se advierte la necesidad de diversificar el repertorio de organizadores en la intervención -integrando además organizadores enumerativos como "en primer lugar", "en segundo término" - y de mostrar a los alumnos que es posible argumentar utilizando otros recursos, como por ejemplo las expresiones condicionales o la inclusión de contra-argumentaciones. 
El trabajo con otro tipo de organizadores se encuentra asociado a la enseñanza y al aprendizaje de las operaciones de apoyo de argumentos, de refutación y negociación. En este sentido, en el marco de la secuencia, se configuraría como un apoyo para el aprendizaje de discursos argumentativos elaborados (GOLDER, 1996).

Signos de Puntuación: El empleo de los signos de puntuación puede incularse con el proceso de organización textual (SCHNEUWLY, 1988). Desde esta perspectiva, y debido a la escasa presencia de signos observada en las producciones iniciales -sólo algunos puntos y en menor medida comas de enumeración-, se decidió introducir en la secuencia actividades sobre el empleo del sistema de puntuación. En las producciones finales se advierten avances. La media de empleo de signos se eleva de 2.32 en la PI a 4.21 en la PF.

Pese a que en la PF se duplica la media de signos, los alumnos utilizan menos puntos de los necesarios. Para dar cuenta de este fenómeno de "subpuntuación", se calculó -siguiendo a Schneuwly (1988) - el porcentaje relativo de puntos, es decir, la cantidad de puntos utilizados en relación con la cantidad de frases en las que los deberían haber utilizado, tomando como referencia el número de unidades terminales en las producciones (HUNT, 1970) ${ }^{4}$. En las producciones iniciales el porcentaje es de 24,29\%; mientras que en las finales se eleva a $37,17 \%$. Aunque el avance es importante, los resultados muestran que los niños están muy lejos de dominar el sistema de puntuación, en tanto recurso que permite organizar y segmentar las unidades de información.

Un aspecto que resulta relevante señalar es que el progreso más marcado en las producciones finales se observa en el empleo de signos ligados al formato de la carta (coma del encabezamiento y dos puntos después del destinatario), lo que se atribuye a un impacto de las actividades de la secuencia. Los estudios sobre adquisición de la puntuación sugieren que el patrón evolutivo sigue un doble proceso de diversificación y estandarización, que alcanza un nivel elevado en tercer grado (FAYOL, 1997; ver revisión en FAVART, 2005). Sin embargo, nada dicen estas investigaciones sobre el papel de la intervención en el proceso. $\mathrm{Si}$, en un tiempo relativamente breve, los niños pueden avanzar en el dominio de signos de puntuación ligados al formato, debido a que las actividades didácticas trabajaron este aspecto, cabe preguntarse si el patrón evolutivo puede acelerarse con la enseñanza. Desde esta perspectiva, nos parece necesario fortalecer la enseñanza de la puntuación en la secuencia.

4 Una unidad sintáctica terminal mínima (UT) es definida como una oración principal más las oraciones subordinadas dependientes de la principal (HUNT, 1970). 
Expresión de la solicitud, modalización y fórmulas de cortesía: Las expresiones de solicitud relevadas permitieron clasificar los textos en tres grupos: textos con a) solicitud directa sin modalizaciones, b) solicitud con alguna marca de modalización, como la presencia del imperfecto de cortesía, el imperfecto del subjuntivo, o el auxiliar poder y c) solicitud expresada con una oración subordinada interrogativa indirecta. Los resultados se presentan en la tabla 3.

Tabla 3. Formulación de la solicitud

\begin{tabular}{|l|c|c|}
\hline Categorías & PI & PF \\
\hline Solicitud directa & $27,4 \%$ & $11,2 \%$ \\
\hline Solicitud con modalización & $35,48 \%$ & $45,1 \%$ \\
\hline Interrogativa indirecta & $20,96 \%$ & $35,4 \%$ \\
\hline
\end{tabular}

El patrón de avance muestra que la distribución de los textos en las categorías se modifica y que se incrementa el empleo de marcas de modalización. La cantidad de cartas con solicitud directa se reduce, y aun en estos textos, se añaden enunciados atenuantes, como subordinadas condicionales: "si usted quiere invitarnos". Asimismo, se incrementan las producciones con algún tipo de modalización y con interrogativa indirecta, aumenta el empleo de frases de cortesía y se amplía el repertorio de fórmulas incluidas. El avance en estos aspectos se relaciona con las actividades orales y escritas desplegadas en el aula.

\subsection{Aspectos transversales}

Tanto en las producciones iniciales como finales se identificaron dificultades ortográficas, problemas para la segmentación de palabras, omisión y trueque de letras en la escritura de palabras y problemas relacionados con la sintaxis. Estos aspectos revelan la incidencia que pueden tener los procesos de nivel inferior tales como la codificación fonológica y la escritura de palabras- sobre la producción textual, cuando dichas habilidades no están aún automatizadas (BERNINGER \& SWANSON, 1994; BRASSART, 1995). Las dificultades que manifiestan los niños evidencian que los procesos de aprendizaje de la escritura no están afianzados, lo que podría influir también sobre otros aspectos de la producción textual como por ejemplo el dominio de las características del género en la escritura. Esto nos conduce a preguntarnos si algunas de las dificultades observadas en el análisis pueden ser atribuidas a problemas para producir los argumentos o para mantener la coherencia o si se limitan a las dificultades para escribir las argumentaciones, debido a la situación de sobrecarga cognitiva que la escritura puede llevar aparejada. En 
trabajos posteriores con secuencias didácticas de género, debería considerarse la introducción de talleres de revisión que aporten estrategias de remediación puntualmente relacionadas con los aspectos transversales.

\section{A MODO DE CONCLUSIÓN}

Este trabajo se propuso explorar los resultados de la aplicación de una secuencia didáctica para andamiar la producción de argumentaciones escritas, en contextos sociales particularmente difíciles. El proceso se presenta como productivo, en tanto que los resultados muestran progresos en todos los alumnos.

La investigación confirma la validez didáctica de una enseñanza precoz de la argumentación escrita. La transposición didáctica del género "carta de solicitud" propuesta en este trabajo no sólo permite un trabajo adecuado con alumnos de primer ciclo de primaria, sino que también clarifica las dimensiones que pueden ser abordadas precozmente y los efectos positivos en el proceso de la escritura. La secuencia didáctica caracterizada por un trabajo sobre a) el formato de la carta, b) la formulación de solicitudes, c) la producción de argumentos y d) la puntuación se revela como un dispositivo didáctico que puede ser utilizado por los docentes de nivel primario, con resultados positivos. Asimismo, las dimensiones elegidas para trabajar son percibidas como coherentes por las maestras participantes, permiten un trabajo sobre la lengua y el discurso y mostraron un efecto globalmente positivo en el desempeño de los estudiantes, como lo muestra el contraste de las producciones iniciales y finales.

En efecto, el análisis de las PI nos permitió conocer y sistematizar el tipo de dificultades en la escritura del género "carta de solicitud". Observamos que los escasos conocimientos sobre el género en cuestión se traducían en dificultades ligadas tanto a la planificación como a la textualización. Los alumnos manifestaban problemas en la adecuación a la consigna - ligada a la situación comunicativa en el aula-, en la organización textual, en el formato convencional de la carta y en el empleo de diferentes recursos textuales.

Luego de la intervención se observaron progresos importantes en las habilidades discursivas de los niños, que se centraron, principalmente, en tres aspectos: la comprensión de la situación comunicativa, el formato de la carta y la planificación. En relación con el primer aspecto, los textos de la producción final pusieron en evidencia que la gran mayoría de los alumnos podía responder a los parámetros comunicativos delimitados en la consigna. Si bien este avance fue 
importante, llaman la atención ciertas características de las producciones que delatan otro tipo de problemas vinculados con la situación comunicativa. Así por ejemplo, la inclusión de contenidos propios de un texto informativo sugiere que los alumnos no comprenden completamente el objetivo de la carta. El carácter "escolar" de la tarea interfiere en la situación de ficcionalización del género de referencia y los alumnos convierten una carta de solicitud para pedir un turno en el zoológico en la descripción, con términos técnicos, de animales. De manera similar, la dificultad para apropiarse de los parámetros argumentativos propuestos en la consigna, que se observan en la producción final sugiere que los problemas se asocian, más bien, a la comprensión de la naturaleza ficcional que es intrínseca a la tarea de escritura escolar (SCHNEUWLY y DOLZ, 1997; VUILLET, MABILLARD, TOBOLA \& DOLZ, en prensa).

Con respecto al formato de la carta, en la producción final prácticamente la totalidad de los alumnos respetaba la estructura convencional del género. Este progreso, que estaría en relación con las actividades desplegadas en el marco de la secuencia didáctica, pone de manifiesto el impacto de la secuencia sobre las producciones de los alumnos. Por otro lado, el formato de la carta -relativamente fácil de aprender (BRASSART, 1995)- parece incidir en la planificación textual.

El tercer avance importante es la manera en la que los niños organizan y estructuran sus textos. En las producciones finales se incrementan los textos con una organización clara y ordenada y se incluyen, además, otros elementos del género, como los agradecimientos o las fórmulas de cierre. En este sentido, las actividades de la secuencia diseñadas para trabajar el formato de la carta parecen haber incidido también en la organización de los textos.

Por otra parte, se observó que los niños pudieron desarrollar también, aunque en menor medida, la planificación argumentativa. Los alumnos, a partir del trabajo con la secuencia didáctica, han avanzado en la posibilidad de apoyar sus demandas con argumentos. La secuencia incide en la organización de los textos, desarrollando el conjunto de los elementos convencionales de una carta argumentativa.

En cuanto a la textualización, los niños progresaron de manera evidente en el empleo de marcas de modalización y de fórmulas de solicitud, en el uso de conectores causales y de signos de puntuación ligados al formato de la carta. Sin embargo, permanecieron las dificultades de puntuación general, la contextualización de la demanda y la relativamente breve cantidad de argumentos incluidos.

En este sentido, es posible observar un correlato evidente entre los avances y los problemas de las producciones finales, por una parte, y la incidencia del dispositivo didáctico por otra. En efecto, los progresos observados se relacionan claramente 
con el tipo de contenidos de los módulos y con las actividades desplegadas en las aulas, puesto que son los aspectos abordados en la secuencia didáctica los que muestran los progresos más importantes en las producciones finales.

Paralelamente, los resultados ponen en evidencia los límites de la implementación de la secuencia en este experimento y la necesidad de continuar la enseñanza basada en el género. Desde la perspectiva del ISD, las producciones iniciales se plantean como un punto de partida que permite identificar las fortalezas de los niños y estimar las dificultades sobre las que habrá que trabajar. La PI constituye un indicador acerca de qué es lo que el niño necesita para avanzar en sus conocimientos del género en cuestión. Por eso, las actividades de la secuencia se diseñan y se aplican en función de lo observado en esa primera fase.

En nuestro estudio, en cambio, las producciones iniciales eran, simplemente, un indicador que permitía medir el avance a partir del contraste con la producción final. La diferencia en el enfoque tuvo, como consecuencia, la desatención de ciertas dificultades identificadas en las producciones iniciales, que se mantuvieron en las producciones finales. En este sentido, resulta clara la necesidad de una implementación crítica de las secuencias. Si bien es cierto que una aplicación masiva de las secuencias didácticas reduce las posibilidades de diseñar instrumentos adecuados para todos los grupos, los resultados positivos de la aplicación del instrumento didáctico dependerán siempre de la consideración de las dificultades reales de los alumnos. Las producciones iniciales se configuran entonces como un factor prioritario para el diseño y la selección de actividades que se pongan en foco, de forma tal que posibilite que los niños avancen en su desempeño lingüístico.

Por otra parte, una dificultad básica de los alumnos fue el desarrollo de las habilidades consideradas de nivel inferior -esto es, la codificación fonológica y el trazado de las letras (BERNINGER \& SWANSON, 1994; BRASSART, 1995; entre otros). Cuando estas habilidades no están lo suficientemente automatizadas, realizan demandas cognitivas al sistema de memoria, demandas que, sumadas a las exigencias de estructuración y planificación textual así como las de la linearización, pueden producir una situación de sobrecarga cognitiva. La sobrecarga puede afectar tanto los procesos de nivel inferior, como los de nivel superior. Nos preguntamos entonces si esta situación de sobrecarga podría explicar la omisión de letras y de palabras detectada en las producciones y al mismo tiempo, si podría tener una incidencia en la organización y planificación textual (SÁNCHEZ ABCHI, BORZONE Y DIUK, 2007, para una revisión). Si bien la automatización de los procesos de nivel inferior resulta básica, en la secuencia se focalizaron fundamentalmente procesos de nivel superior. En futuros estudios nos parece necesario considerar el impacto que las 
actividades o las instancias de revisión que atiendan a los procesos de nivel inferior pueden tener en las producciones de los más pequeños.

Pese a los límites del dispositivo, los avances generales reafirman, por una parte, la posibilidad de trabajar el texto argumentativo desde edades tempranas $y$, por otra, el impacto que pueden tener las secuencias didácticas en el desempeño de los niños. Desde una perspectiva vigotskyana, el trabajo con géneros textuales, a partir de secuencias didácticas, permite la progresión y la articulación de los contenidos y promueve los aprendizajes en producción escrita de los alumnos. En resumen, la experiencia pone de manifiesto la teoría de los géneros didácticos aplicada a la escuela (SCHNEUWLY \& DOLZ, 2004).

\section{REFERENCIAS BIBLIOGRÁFICAS}

N. A. P. Núcleos de Aprendizaje Prioritario. Nivel Inicial y Primer ciclo de la E. G. B. (2004). Disponible en: $\quad<$ http://www.educaciencias.gov.ar/archivos/nap/nap-egb-primario.pdf $>$ Acceso el 2 de octubre 2012.

CBC. Contenidos Básicos Comunes de la Educación General Básica de la República Argentina. (1997). Buenos Aires: Ministerio de Cultura y Educación de la Nación.

ARROSSI, F.; AXELRUD, B.; D'AGOSTINO, M. \& EISNER, L. (2002). Competencias argumentativas en alumnos universitarios. En: García Negroni, M. (ed.) Actas del Congreso Internacional La Argumentación. Buenos Aires: UBA, pp.656-664.

BEREITER, C. \& SCARDAMALIA, M. (1987). The psychology of written composition. Hillsdale, NJ.: Lawrence Erlbaum.

BERNINGER, V. \& SWANSON, L.(1994). Modifying Hayes and Flower's Model of Skilled writing to explain beginning and developing writing. Advances in cognition and educational practice. Vol. 2, pp. 57-81.

BRASSART, D. (1995). Elementos para una didáctica de la argumentación en la escuela primaria. Comunicación, Lenguaje y Educación. $\mathrm{N}^{\circ}$ 7, pp. 41-50.

BRONCKART, J. P. (2004). Actividad verbal, textos y discursos. Por un interaccionismo socio-discursivo. Madrid: Fundación Infancia y Aprendizaje.

BRONCKART, J. P.; BAIN, D.; SCHNEUWLY, B.; DAVAUD, C. \& PASQUIER, A. (1985). Le fonctionnement des discours. Paris- Neuchâtel: Delachaux \& Niestlé.

CAMPS, A. (2003). (comp.) Secuencias didácticas para aprender a escribir. Barcelona: Grao.

CAMPS, A. \& DOLZ, J. (1995). Enseñar a argumentar: un desafío de la escuela actual. Comunicación, Cultura y Lenguaje. $\mathrm{N}^{\circ} 25$, pp. 5-8.

COTTERON, J. (1995). ¿Secuencias didácticas para enseñar a argumentar en la escuela primaria? Comunicación, Lenguaje y Educación. $\mathrm{N}^{\circ}$ 25, pp. 79-94. 
DOLZ, J. (2001). Tâches et outils dans des nouveaux moyens d'enseignement sur l'argumentation: analyse contrastive de la progression à l'oral et à l'écrit. En Les tâches et leurs entours en classe de français: actes du se Colloque international de la DFLM, Neuchâtel, septembre 2001. Neuchâtel: IRDP (Artículo en CD-ROM).

DOLZ, J. (1996). Learning argumentative capacities. A study of the effects of a systematic and intensive teaching of argumentative discourse in 11-12 year old children. Argumentation. $\mathrm{N}^{\circ} 10$, pp. 227-251.

. (1995). Escribir textos argumentativos para mejorar su comprensión, Cultura y Educación. $\mathrm{N}^{\circ} 25$, pp. 65-77.

DOLZ, J., GAGNON, R. \& TOULOU, S. (2008). Production écrite et difficultés d'apprentissage. Genève: Carnets des Sciences de l'éducation. Diptyque. n 13, pp. 43-92.

DOLZ, J., MABILLARD, J.P., TOBOLA COUCHEPIN, C. \& VUILLET, Y. (2009). Analyse contrastée des difficultés des élèves dans la rédaction d'une réponse au courrier des lecteurs, et de leur traitement en classe. Revue suisse de sciences de l'educaction. $\mathrm{N}^{\circ} 3$, pp. 41-57.

DOLZ, J.; NOVERRAZ, M. \& SCHNEUWLY, B. (2001). S'exprimer en français, vol I-IIIII-IV, De Bruselas: Boeck.

DOLZ J. \& PASQUIER, A. (2000). Escribo mi opinión. Fondo de Publicaciones del Gobierno de Navarra.

. (1993a). Argumenter... pour convaincre: initiation aux textes argumentatifs, Cabiers du Service du Français, 31, Genève: Direction d'instruction publique (DIP).

(1993b), «Mon avis, je l'écris»: initiation aux textes d'opinion, Cabiers du Service $d u$ Français, 35, DIP, Genève.

DOLZ, J. \& SCHNEUWLY, B. (1996). Apprendre à écrire ou comment étudier la construction de capacités langagières? Etudes de Linguistique Apliquée. 101.

FAVART, M. (2005). Les marques de cohésion: leur rôle fonctionnel dans l'acquisition de la production écrite de texte.Psychologie Française, 50,3, pp. 305-322.

FAYOL, M. (1997). On acquiring and using punctuation: A study of written French. In Jean Costermans and Michel Fayol, (eds.) Processing Interclausal Relationships: Studies in the Production and Comprebension of Text, Mahwah, NJ: Lawrence Erlbaum Associates.

FAYOL, M., \& SCHNEUWLY, B. (1988). La mise en texte et ses problèmes. In J.L. Chiss, J.P. Laurent, J.C. Meyer, H. Romian, \& B. Schneuwly (Eds), Apprendre/enseigner à produire des textes écrits. Bruxelles: Duculot.

FELTON, M. (2004). The development of discourse strategies in adolescent argumentation. Cognitive Development. №19, pp. 35-52.

GARCÍA JURADO, M., LÓPEZ GARCÍA, M. \& MIÑONES, L. (2002). Construcciones Concesivas en producciones escritas de alumnos pre universitarios. Ed. García Negroni, M. Actas del Congreso Internacional La Argumentación. Buenos Aires. UBA. pp. $725-732$. 
GOLDER, C. (1996). Le développement des discours argumentatifs. Lausanne: Delachaux \& Niestlé. HOCEVAR, S. (2007). La génesis de la argumentación. Comunicación presentada en las Primeras Jornadas de lectura y escritura. Lectura y escritura críticas: perspectivas múltiples. San Miguel de Tucumán, Argentina.

HUNT, K. W. (1970). Recent measures in syntactic development. In Lester, M. (ed.) Reading in applied transformational grammar. Nueva York: Holt, Rinehart and Wiston, pp. 179- 192.

IDIAZABAL, I. \& LARRINGAN, L. (1997). Transfert de maîtrises discursives dans un programme d'enseignement bilingue basque-espagnol Acquisition et Interaction en Langue Étrangère. $\mathrm{N}^{\circ} 10, \mathrm{pp} .107-125$.

KUHN, D. \& UDELL, W. (2003). The development of argument skills. Child Development, Vol. 74, No. 5, pp. 1245-1260.

NUÑEZ LAGOS, P. (1999). El desarrollo de la competencia textual argumentativa y sus implicancias en la sala de clases. Revista Lingüística en el aula. №3, pp. 41-58.

PARODI SWEIS, G. (2000). La evaluación de la producción de textos escritos argumentativos: una alternativa cognitivo/discursiva. Revista Signos. V. 33, pp. 151 166.

PIETRO, J. F., PFEIFFER RYTER, V.; WIRTHNER, M.; BEGUIN, M.; BROI, A. M.; CLÉMENT, S.; MATEI, A. \& ROOS, E. (2009). Evaluation de Moyen d'enseignement S'exprimer en francais. Rapport Final. Neuchâtel: Institut de Recherche et docummentation pedagogique.

SAINZ OSINAGA, M. (2001). Azalpenezko testu entziklopedikoaren azterketa eta didaktika. Tesis Doctoral Inédita. Donostia: Erein.

SÁNCHEZ ABCHI, V. (2009). El aprendizaje de la escritura en niños pequeños de medios rurales y urbanos. Un estudio de los subprocesos de transcripción y generación textual. Tesis doctoral Inédita. Universidad Nacional de Córdoba.

SÁNCHEZ ABCHI, V., BORZONE, A. M. \& DIUK, B. (2007). La escritura de textos en niños pequeños: relación entre la transcripción y la composición, Universitas Psicológica. V. 6, $\mathrm{N}^{\circ} 3$, pp. 559-570.

SÁNCHEZ ABCHI, V. \& BORZONE A. M. (2010). El aprendizaje de la escritura en niños pequeños de medios rurales y urbanos. En Viramonte de Avalos, M. (Dir.) Saludy aprendizajes lingiǘsticos. Complejidades en la enseñanza de la lengua. Córdoba, Argentina: Ed. Comunicarte.Tomo 2, pp. 245-278.

SANTAMARIA, J. (2003). Escribir textos argumentativos: una secuencia didáctica. En Camps, A. (comp.) Secuencias didácticas para aprender a escribir. Barcelona: Grao. pp.111126.

SCHNEUWLY, B. (1988). Le langage écrit chez l'enfant La production des textes informatifs et argumentatifs. Neuchâtel: Delachaux et Niestlé.

SCHNEUWLY, B. \& DOLZ, J. (1997). Les genres scolaires des pratiques langagières aux objets d'enseignement. Repères. No 15, pp.27-40. 
SCHNEUWLY, B. \& DOLZ, J. (2004). Gêneros orais e escritos na escola. Campinas, SP: Mercado de Letras.

SILVA, M.L. (2002). El desarrollo de las estrategias de argumentación: aportes y observaciones a partir del análisis de un caso. Actas del Congreso Internacional "La Argumentación" Buenos Aires: Fac. de Filosofía y Letras. pp. 101-115.

SILVESTRI, A. (2001). La producción de la argumentación razonada en el adolescente: las falacias de aprendizaje. En Martínez, M.C. (comp.) Aprendizaje de la argumentación razonada. Cali: Cátedra UNESCO para la lectura y la escritura en América Latina.

VUILLET, Y., MABILLARD, J. P., TOBOLA, C. \& DOLZ, J. (en prensa) Dédoublement du genre et des opérations de fictionnalisation, comment faire «comme si c'était vrai »? Education et didactique. Rennes: PUR.

VIGOTSKY, L.S. (1988). El desarrollo de los procesos psicológicos superiores. Barcelona: Grijalbo.

Recebido: 01/09/2011

Aceito: 09/10/2012 